\title{
Covid-19 Pandemic: Cardiovascular and Neurologic Im- pact, Early Signs and Symptoms
}

\author{
Juan Carlos Chachques ${ }^{1 *}$, Letizia Mazzini², Dinko Mitrecic $^{3}$, Christian Hellmich ${ }^{4}$, Barbara \\ Zavan $^{5}$, Massimo Rogante ${ }^{6}$, Christian Latremouille ${ }^{1}$ and Franco Rustichelli ${ }^{7}$ \\ ${ }^{1}$ Cardiovascular Division, Pompidou Hospital, France
}

${ }^{2}$ Department of Neurology, Maggiore della Carità Hospital, Italy

${ }^{3}$ Croatian Institute for Brain Research, School of Medicine University of Zagreb, Croatia

${ }^{4}$ Vienna University of Technology (TU Wien), Austria

${ }^{5}$ Department of Medical Sciences, University of Ferrara, Italy

${ }^{6}$ Rogante Engineering Office, Italy

${ }^{7}$ Physical Sciences Division, Polytechnic University of Ancona-Marche, Italy

*Corresponding author: Juan Carlos Chachques, Cardiovascular Division, Pompidou Hospital, University of Paris. 56 rue Leblanc, 75015 Paris, France.

To Cite This Article: Juan Carlos Chachques, Letizia Mazzini, Dinko Mitrecic, Covid-19 Pandemic: Cardiovascular and Neurologic Impact, Early Signs and Symptoms. 2020 - 11(1). AJBSR.MS.ID.001586. DOI: 10.34297/AJBSR.2020.11.001586.

Received: 眥 November 12, 2020; Published: 眥 November 30, 2020

\begin{abstract}
Background: Scientists and physicians continue their cutting-edge work on bacteria, parasites and viruses, responsible for 17 million deaths each year worldwide. The purpose of this article is to review our clinical experience with COVID-19 patients observed in the Cardiovascular Division of Pompidou Hospital (University of Paris, France) and the Department of Neurology of the Eastern Piedmont University (Novara, Italy), related with the impact on the cardiovascular, hematologic, neurologic systems and sense organs. Mathematical modeling for pandemic spreading control (protection versus hazards), proposed by Vienna University of Technology (Austria), are analyzed.
\end{abstract}

Methods: We sought to characterize the cardiovascular and neurologic manifestations, in patients with Covid-19. Special attention was given to initial signs and symptoms in order to facilitate early diagnosis and therapy. Indications of ECMO membrane oxygenation for cardio-respiratory support are evaluated, mathematical models for control pandemic spreading are described.

Results: Early neuro-sensorial symptoms like anosmia and dysgeusia are useful for diagnostic, patient's isolation, and treatment. Angiohematologic acro-ischemia syndrome and dermatological signs are mostly related with immune dysregulation, neoangiogenesis, vasculitis, and vessel thrombosis resulting from "cytokine storm syndrome". Acro-ischemia syndrome allows diagnosis and early treatment of disseminated intravascular coagulation, with mortality risks for children and adults.

Conclusion: Covid-19 is a prothrombotic disease with a unique global lethality. Strong inflammatory response to viral infection severely affects cardiovascular, and neurologic systems, as well as respiratory, immune and hematologic systems. Fast identification of acro-ischemia syndrome permits the care of disseminated intravascular coagulation complications. Early symptoms like gustatory and olfactory loss are useful for Covid diagnostic. Mathematical models play a significant role in the understanding of COVID-19 transmission mechanisms, structures, and features. It should be of interest for policymakers and for explaining recurrent epidemic outbreaks.

Abbreviations: COVID $\quad$ : Corona Virus Disease; SARS CoV $\quad$ : Severe Acute Respiratory Syndrome Coronavirus; ECMO: Extra Corporeal Membrane Oxygenation; ACE: Angiotensin Converting Enzyme; RT-PCR : Real Time - Polymerase Chain Reaction; PEEP: Positive EndExpiratory Pressure; ARDS: Acute Respiratory Distress Syndrome; HFNC: High Flow Nasal Cannula; NIV: Non-Invasive Ventilation; L/min: Liter per Minute; HEPA: High Efficiency Particulate filter; CMR: Cardiac Magnetic Resonance; DVT: Deep Venous Thrombosis; MERS-CoV: Middle East Respiratory Syndrome Coronavirus; D-DIMER: Fibrin Degradation Product; ICU: Intensive Care Unit; DIC: Disseminated Intravascular Coagulation; CNS: Central Nervous System; CT: Computed Tomography; LU: Lung Ultrasound; SEIR Epidemiology model: Susceptible-Exposed-InfectiousRecovered 
Keywords: Covid-19 pandemic, Cardio-vascular \& Neuro-sensorial impact, Acro-ischemia Syndrome, Disseminated intravascular coagulation, Anosmia, Dysgeusia, Cytokine storm syndrome, Ecmo membrane oxygenation, Synchrotron radiation diffraction

\section{Background}

COVID-19 caused by coronavirus 2 (SARS-CoV-2) has been declared a pandemic by the World Health Organization on March 11, 2020 [1]. After 3 months of critical clinical situation in Western Europe, the number of Covid cases, severity and mortality rates showed optimistic parameters. However, progressively coronavirus returns to Europe in September 2020 (Figure 1). As of 4 November 2020, COVID-19 has led to over 47.8 million confirmed infections and 1,220,224 deaths from coronavirus disease-2019 worldwide. Like most infections caused by members of the coronavirus family, SARS-CoV-2 manifests itself with upper respiratory tract infections and flu-like symptoms of varying severity. However, Covid-19 is unique in its ability to cause a multi-organ disease, with involvement of the cardiovascular and nervous systems. Coronavirus can send the body's immune system into cataclysmic overdrive, and cause blood thrombosis that impede circulation to lungs, heart, brain, or limbs [2-4].

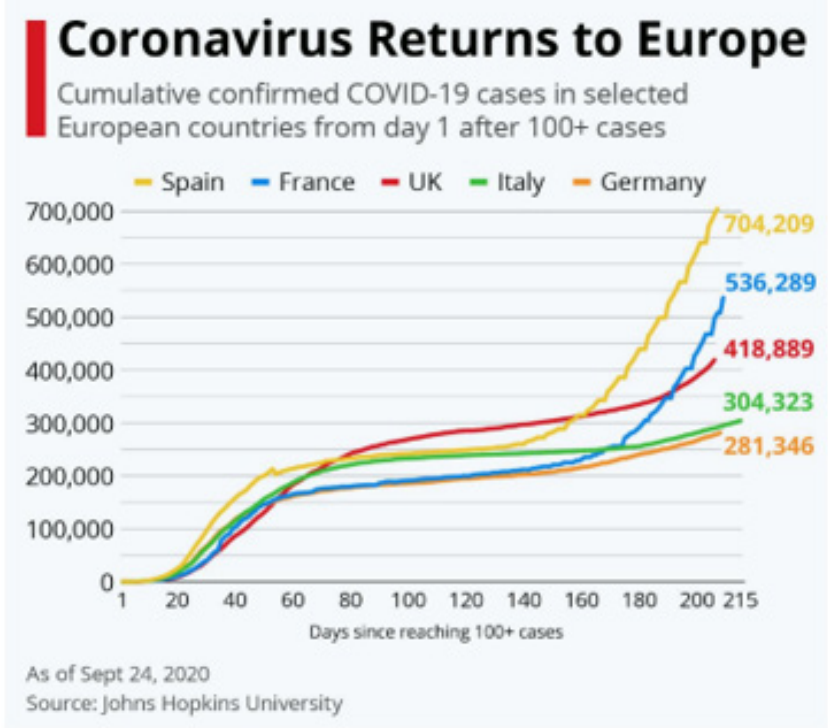

Figure 1: Cumulative confirmed COVID 19 cases in 5 selected European countries (as of September 24, 2020).

The main goal of this article is to review our clinical experience observed with COVID-19 patients during the 2020 pandemic in the Cardiovascular Division of Pompidou Hospital (University of Paris, France) and the Department of Neurology of the Eastern Piedmont University (Novara, Italy). It analyses the impact on the cardiovascular, hematologic, neurological systems and initial signs and symptoms of Covid-19. Additionally, we present mathematical models proposed by Vienna University of Technology (Austria), for control pandemic spreading (protection versus hazards).

\section{Covid-19, from SARS to Multiorgan Disease}

Although Severe Acute Respiratory Syndrome (SARS) is at the core of the disease, covid-19 has revealed itself to be more than a simple viral pneumonia. SARS-CoV-2 disease seriously affects respiratory, cardiovascular, neurologic, and immune systems [5-7]. The three most common initial symptoms in COVID are fever, dry cough, and shortness of breath. Patients can also suffer from mus- cle pain, sore throat, nasal congestion, headache, loss of smell and taste and persistent hiccups. Current clinical experience is showing that it is important to monitoring blood oxygen saturation using pulse oximetry devices to indicate mask oxygen therapy. Non-invasive ventilation is recommended. Barotrauma in mechanical ventilation can be observed creating rupture of the alveolus $[8,9]$. Characteristics of COVID patients who become seriously ill are different of classic "pneumonia" or ARDS (Acute Respiratory Distress Syndrome). Coronavirus causes prolonged and progressive hypoxia, desaturation, leading to organ failure and fatal outcome. Thrombolytic therapy in COVID-19 patients with ARDS may be of benefit due to the unusually high incidence of pulmonary embolism and pulmonary thrombosis, particularly microvascular thrombosis, which are thought to contribute significantly to hypoxemia. It may also ameliorate the effects of extravascular and intra-alveolar fibrin deposition described in ARDS [10-12]. 
Covid-19 patients can remain subjectively comfortable even when their oxygen saturation levels fall far below borderline ranges, becoming a "silent hypoxemia", low oxygen saturation associated with hypocapnia can be observed in pulmonary artery embolism [13]. Thoracic CT angiography scan and lung ultrasound imaging provide information for COVID diagnose and follow-up [14,15].

\section{Cardiovascular and Hematological Impact}

Covid-19 can have fatal consequences for people with underlying cardiovascular disease $[7,16,17]$. Over 65 years-old, patients with coronary artery disease, arterial hypertension and cardiac arrythmias are at more severe risk. More worrisome is the fact that COVID could cause heart damage in patients who did not have any previous problems [7]. It is reasonable to expect that significant cardiovascular complications from Covid-19 will occur in patients with severe symptoms due to the strong inflammatory response associated with this disease that affects the myocardium. The critical cases of Covid-19 are those which have reported respiratory failure, septic shock with evolution towards multiorgan failure.

In a new study including 100 patients recently recovered from COVID-19 infection [18], cardiovascular magnetic resonance imaging (CMR) revealed cardiac involvement in 78 patients (78\%) and ongoing myocardial inflammation in 60 patients $(60 \%)$, independent of pre-existing conditions, severity and overall course of the acute illness, and time from the original diagnosis. These findings indicate the need for ongoing investigation of the long-term cardiovascular consequences of COVID-19.

\section{Viral Myocarditis}

$1 \%$ to $5 \%$ of all patients with acute viral infections may involve the myocardium [19]. The incidence of myocarditis is between 10 to 22 cases per 100,000 persons, it was observed in many viruses including enterovirus coxsackie B, parvovirus B-19, herpesvirus, influenza virus, and Covid-19 [20]. Viral myocarditis symptoms can range from nonspecific fatigue and shortness of breath to more aggressive symptoms that mimic acute coronary syndrome. After the initial acute phase, remission of symptoms can be observed or the viral infection may persist, leading to a persistent autoimmune-mediated inflammatory process with continuing symptoms of heart failure. Fulminant myocarditis associated with influenza A (H1N1) virus were observed in previously healthy patients [21]. Treatments include drugs, cardiac bio-assist [22,23], mechanical circulatory assist devices or heart transplantation [24].

\section{Hematology}

Clinical patient's outcome allows to consider COVID-19 as a new hematologic disease, thrombosis would be favored by the vascular attacks related to the Covid-19 infection. Curative anticoagulation in these patients prevents endothelial lesions. Angiopoietin-2 as a marker of endothelial activation is a good predictor factor for in- tensive care unit (ICU) admission [25-28]. Pulmonary artery and vein thrombo-embolism doubled among ICU patients. Endotheliopathy, the activation of coagulation and the inflammatory syndrome contribute to increasing the risk of thrombosis, causes seem to be multifactorial. In obese patients, there is also impaired ventilation which can affect inflammation and organs perfusion. Elevated levels of D-dimers and clotting factors are known risk factors for pulmonary embolism in these cases [10]. COVID-19 seems to be an additional risk factor for Deep Vein Thrombosis (DVT). The prevalence of DVT in hospitalized patients with SRAS-CoV-2 infection is high and is associated with adverse outcomes [29].

\section{Pathological Findings}

Autopsies around the world show that the lungs of the COVID-19 had hundreds of micro clots inside the pulmonary vasculature, not observed in classic pneumonia. In some cases, migrate causing myocardial ischemia or brain stroke. In 38 autopsies analyzed in North Italy [30], the predominant pattern of lung lesions in COVID-19 patients was diffuse alveolar damage, as previously described for the other two coronavirus that infect humans, SARS$\mathrm{CoV}$ and MERS-CoV. Hyaline membrane formation and pneumocyte atypical hyperplasia were frequently found. The main relevant finding was the presence of platelet-fibrin thrombi in small arterial vessels; this important observation fits into the clinical context of coagulopathy which dominates in these patients and which is one of the main targets of therapy [10-13].

Extensive histopathological analysis [31] showed that SARSCoV-2 infection can result in diverse, multi-organ pathology, the most significant being in the lungs (diffuse alveolar damage in its different phases, microthrombi, bronchopneumonia, necrotizing bronchiolitis, viral pneumonia), heart (lymphocytic myocarditis), kidney (acute tubular injury), central nervous system (microthrombi, ischemic necrosis, acute hemorrhagic infarction, congestion, and vascular edema), lymph nodes (hemophagocytosis and histiocytosis), bone marrow (hemophagocytosis) and vasculature (deep vein thrombosis).

\section{Role of ECMO in Covid-19 infection}

Extracorporeal Membrane Oxygenation has been proposed in the treatment of severe pulmonary and cardiac compromise in COVID-19 [32]. ECMO is an extracorporeal respiratory assistance system, its indications concern patients with severe acute respiratory distress syndrome (ARDS) for which all other approaches have failed. There are 2 indications for ECMO: A) can serve as pure respiratory assistance, the blood is then taken from the right side at the level of the inferior vena cava and is reinjected into the upper vena cava. The blood passes through an artificial membrane, which allows complete extracorporeal respiration with oxygenation and decarboxylation of the blood. B) ECMO machine acts as an artificial 
heart-lung, with additional cardiac support. The blood is reinjected this time, not into the jugular vein, but into the aorta $[33,34]$. The care under ECMO can last several weeks. For influenza ARDS patients who are managed every winter, the ECMO is used over an average of 2-3 weeks. For Covid-19 patients, some of them need 1 month of care (Figure 2).

A

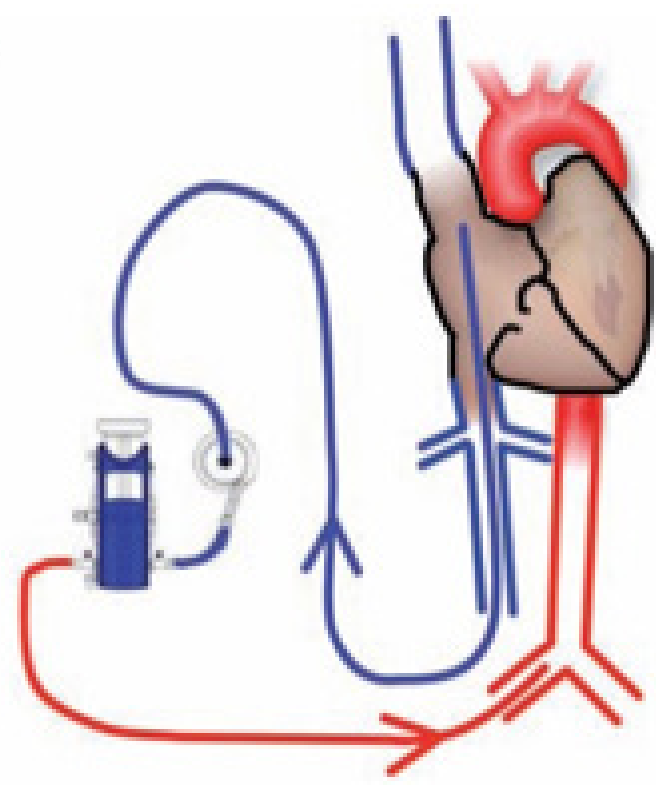

B

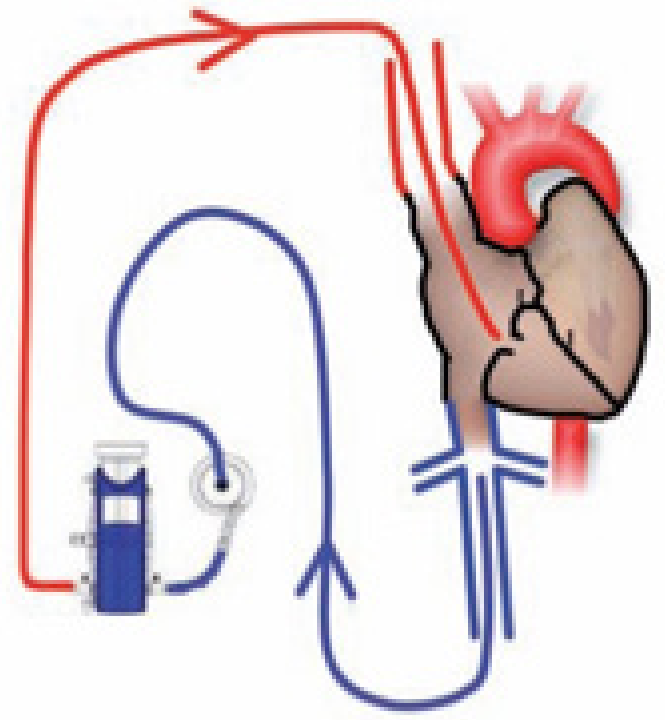

Figure 2: ECMO (Extracorporeal Membrane Oxygenation). ECMO machine is connected to patients through cannula, placed in large veins in the legs, neck or chest, and pumps blood from the patient's body to an artificial lung (oxygenator) that adds oxygen to it and removes carbon dioxide. The ECMO machine then sends the blood back to the patient via a pump with the same force as the heart, to arteries (A) or veins (B).

\section{Acro Ischemia Syndrome}

The basement membrane of the skin is equal to the renal membrane, the ocular structures, etc., and it receives the same type of aggression by immunoglobulins, immunocomplexes and all inflammatory cascades [35]. Acro-ischemia syndrome can be observed in Covid-19 children and adults requiring ICU hospitalization. This syndrome includes toe and/or finger cyanosis, skin bulla and dry gangrene. D-dimer, fibrinogen, and fibrinogen degradation product (FDP) are frequently elevated, increasing progressively when Covid exacerbated (Figure 3). Most of these patients are diagnosed with disseminated intravascular coagulation (DIC), receiving anticoagulation therapy (low molecular weight heparin). The association of respiratory and cardiovascular complications leads to a fatal prognosis [36]. Therefore, the existence of hypercoagulation status in critical Covid patients should be closely monitored. 

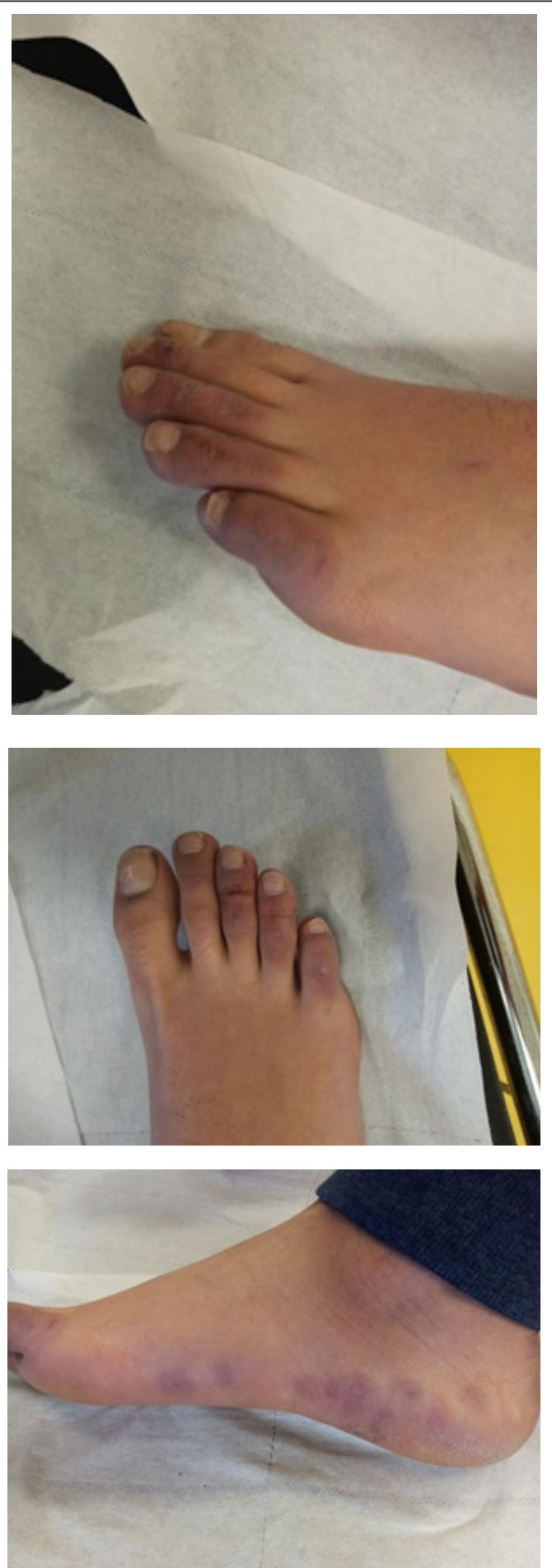

Figure 3: Acro-ischemia syndrome. Cutaneous small vessel vasculitis secondary to COVID-19 infection. Presentation signs include cyanosis on the fingers/toes, sole and heel, skin bulla and dry gangrene. In these cases, D-dimer, fibrinogen, and fibrinogen degradation product (FDP) are significantly elevated, this is related with disseminated intravascular coagulation (DIC).

Dermatological lesions are observed in the face, hands and feet, like "frostbite", including redness at the level of the fingers, nose and ears, swelling, temporary pain which corresponds to what it is observed in winter sports. In addition to this pseudo-frostbite, other skin manifestations have been identified, such as the sud- den onset of persistent, sometimes painful redness, and urticarial eruptions (Figure 4A-4D). The pathophysiology of these lesions is unclear but may include immune dysregulation, vasculitis, vessel thrombosis and neoangiogenesis $[37,38]$. 


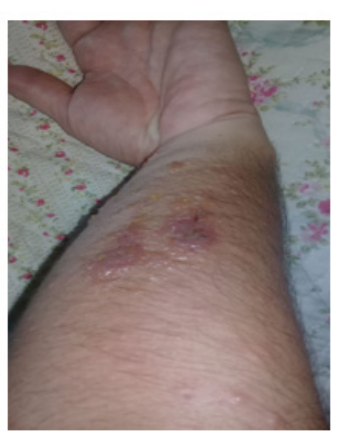

Figure 4A

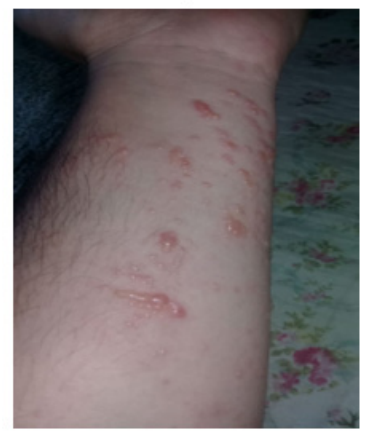

Figure 4B

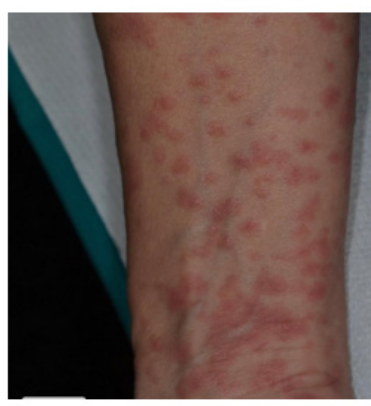

Figure 4C

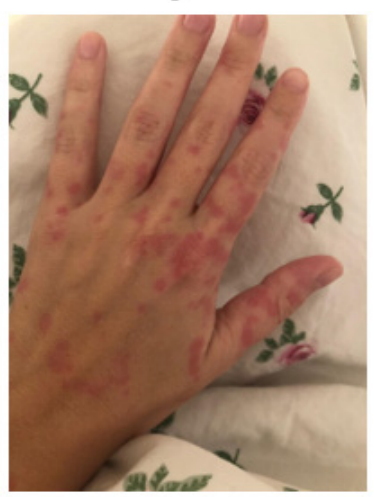

Figure 4D

Figure 4A-4D: Initial dermatological signs of COVID-19. Small vessel vasculitis: hives (urticaria), generalized eruptions (rashes), redness, perniosis (childblains), violaceous macules, non-necrotic purpura.

\section{Impact on Neurological System}

Neurological involvement in Covid-19 [39,40], 0with expression in the most serious patients, can be divided into three categories: a) Symptoms of central neurological concern (such as headache, dizziness, syncope, consciousness alteration, ataxia)

b) Symptoms of neuro-peripheral origin (hypo-ageusia, hyposmia, neuralgia, periphearl neuropathies) 
c) Symptoms of peripheral-muscular damage, often associated with the most critical stages of the disease, with liver and kidney suffering

To support the clinical suspicion of neurological involvement, it is already known that human coronaviruses can spread from the respiratory tract to the central nervous system (CNS) through transneuronal and hematogenous routes, resulting in encephalitis and other neurological complications. Moreover, has been hypothesized that Covid-19, similarly to other coronavirus can spread via a synapse-connected route to the medullary cardiorespiratory center from the mechanoreceptors and chemoreceptors in the lung and lower respiratory airways triggering the acute respiratory failure in infected patients [41]. Another important observation is that $\mathrm{HCoV}$ OC43 RNA, a kind of human coronaviruses, could be detected for at least a year in the CNS of infected mice. Hence If the SARS-CoV-2 is latent in CNS for a long-time, neurological disease can manifest as possible late complications of the cured patients [42].

A wide range of neurologic manifestations of SARS-CoV-2 infection have been recognized, and evidence of their severity and persistence is increasing. Neurologic manifestations were analyzed in 509 consecutive patients admitted with confirmed Covid-19 within a hospital network (Chicago, Illinois) [43]. Neurologic manifestations were present at Covid-19 onset in 215 (42\%), at hospitalization in 319 (62\%), and at any time during the disease course in 419 patients (82\%). The most frequent neurologic manifestations were myalgias (44\%), headaches (37\%), encephalopathy (31\%), dizziness (29\%), dysgeusia (15\%), and anosmia (11\%). Strokes, movement disorders, motor and sensory deficits, ataxia, and seizures were uncommon ( 0.2 to $1.4 \%$ of patients each). Severe respiratory disease requiring mechanical ventilation occurred in 134 patients $(26 \%)$. Independent risk factors for developing any neurologic manifestation were severe Covid-19 and younger age. Of all patients, 362 (71\%) had a favorable functional outcome at discharge. However, encephalopathy was independently associated with worse functional outcome and higher mortality within 30 days of hospitalization. In summary, neurologic manifestations occur in most hospitalized Covid-19 patients. Encephalopathy was associated with increased morbidity and mortality, independent of respiratory disease severity.

The clinical neurological practices have, by necessity, changed dramatically during the pandemic spreading. Patients with acute, non-infectious, neurological disorders, that it is means, for almost all cases, patients with ischemic or hemorrhagic vascular events, are tending to postpone the access to the emergency care for several reasons, such as the contagion fear or the anxiety of subsequent isolation from the family unit, or to avoid a further overloading of national health service. However, the delay in access leads to an impossibility of early essential treatment with an inevitable wors- ening of the long-term outcome. On the other hand, the approach and needs of patients with chronic neurological diseases (e.g. epilepsy or multiple sclerosis) and of patients with neurodegenerative diseases (e.g. Alzheimer's disease and other forms of progressive dementia, Parkinson's disease and Amyotrophic Lateral Sclerosis are inevitably different. In this worldwide situation, where neurological diseases do not stop only because a pandemic is ongoing, the switch to non-face-to-face care, is becoming mandatory to prevent the physical and psychological consequences that can be associated with this emergency [44]. Various telehealth technologies, in particular video teleconferencing between healthcare providers and patients, have been incorporated into the practice of neurology [45].

\section{Loss of Sense of Smell and Taste as Viral Infection Mark- ers}

Early single reports and surveys have suggested that gustatory and olfactory loss may be early symptoms associated with COVID-19 infection also in the absence of other known symptoms of the disease. The first large Multicenter European Study [46] reported that in a population of 417 mild-to-moderate COVID-19 patients $85 \%$ and $88 \%$ had smell and taste dysfunctions respectively with a significant positive association between the two symptoms. Interesting the symptoms were persistent in $56 \%$ of patients over the days following the resolution of the infection general clinical manifestations. Smell and taste loss were reported in $68 \%$ and $71 \%$ of Covid-19-positive subjects in US. Anosmia, with or without dysgeusia, seems to manifest either early in the disease process or in patients with mild or no constitutional symptoms. According to the results of these studies the prevalence of olfactory and gustatory dysfunction seems lower in Asiatic than in European and American COVID-19 patients.

Post-viral anosmia is one of the leading causes of loss of sense of smell in adults, accounting for up to $40 \%$ cases of anosmia. Viruses that give rise to the common cold are well known to cause post-infectious loss, and over 200 different viruses are known to cause upper respiratory tract infections. Previously described coronaviruses are thought to account for $10-15 \%$ cases. It is therefore perhaps no surprise that the novel COVID-19 virus would also cause anosmia in infected patients. However, the traditional nasal cavity manifestations, such as nasal congestion or rhinorrhoea are commonly absent in patients with COVID-19 suggesting a direct SARS-CoV-2 actions in the nervous system, especially in the olfactory pathway. Most studies suggest that the nasal cavity olfactory epithelium is the likely site of enhanced binding of SARS-CoV-2 and that multiple non-neuronal cell types present in the olfactory epithelium express two host receptors, ACE2 and TMPRSS2 proteases, that facilitate SARS-CoV-2 binding, replication, and accumulation. Hence the olfactory epithelium from the nasal cavity has been sug- 
gested as the more appropriate tissue for detection of SARS-CoV-2 virus at the earliest stages. The olfactory receptor neurons may initiate rapid immune responses at early stages of the disease and viruses may first invade peripheral nerve terminals, and then gain access to the CNS via a synapse-connected route. The chance of brain infection through olfactory bulb during COVID-19 infection should be also considered. Clinical evidence of the association of COVID-19 infection and encephalopathy have been reported. Moreover, it is currently suspected that the neuroinvasive potential of SARS-CoV2 plays a key role in the respiratory failure of COVID-19 patients $[47,48]$. These preliminary observations suggest that research should be focused on additional aspects of SARS-CoV-2 actions in the olfactory pathway.

Mathematical Modeling to Control Epidemic Spreading: Protection Versus Hazards (Polytechnic University of Vienna, Austria)

Mathematical models address the need for understanding the transmission dynamics and other significant factors of the disease that would aid policymakers to make accurate decisions and reduce the rate of transmission of the disease.

The multi-disciplinary fight against the global spreading of COVID-19 has a strong applied mathematics/engineering science component. The outbreak of COVID-19 put in evidence the huge positive potential of diligent and careful use of applied mathematics. In this context, stochastic transmission models [49], also in combination with digital twin approaches, have turned out to be extremely helpful for estimating the efficiency of different approaches for well-tailored partial or full lock-down scenarios. In countries such as Singapore or Austria, characterized by comparatively low mortality of these Asian or European regions, governmental lockdown decisions were in fact strongly supported by such mathematical approaches.

The reliability of non-modified classical epidemiological models, i.e. compartmental model of the SEIR-type, has turned out as very questionable. Such models have repeatedly delivered predictions which, just a few days or weeks later, turn out to lie far away from the recorded data on new infections or new deaths. The basic reason for this situation seems to be in the actual non-identifiability of the involved model parameters [50]. This calls not only for simple model structures focusing on the key characteristics of the pandemic spreading, but also for careful modification of the compartmental model-related differential equations themselves. These mathematical models play a significant role in the understanding of COVID-19 transmission mechanisms, structures, and features. This may pave the way to new coronavirus spreading models - namely to such models which might help us to successfully fight COVID-19, while still seeing a beautiful, "simple", mathematical structure tell- ing us analytically where the weaknesses of the virus could be [5153].

\section{Bioengineering Developments for Diagnostic and Therapy}

\section{Non-Invasive Ventilation (NIV) in SARS}

High Flow Nasal Cannula (HFNC) and Facemask are in development for severe acute hypoxemic respiratory failure of COVID-19 patients [54]. Instead of mechanical ventilation, a recent clinical study [55] showed that HFNC, which targeted a flow $\geq 50 \mathrm{~L} / \mathrm{min}$, improves oxygenation, reduces minute ventilation, and work of breathing. This study (performed in 379 patients) demonstrated clinical benefits, reduced patients' intubation, and subsequent invasive mechanical ventilation. Thus, HFNC seems to be as safe as standard oxygen in COVID-19 patients (Figure 5A).

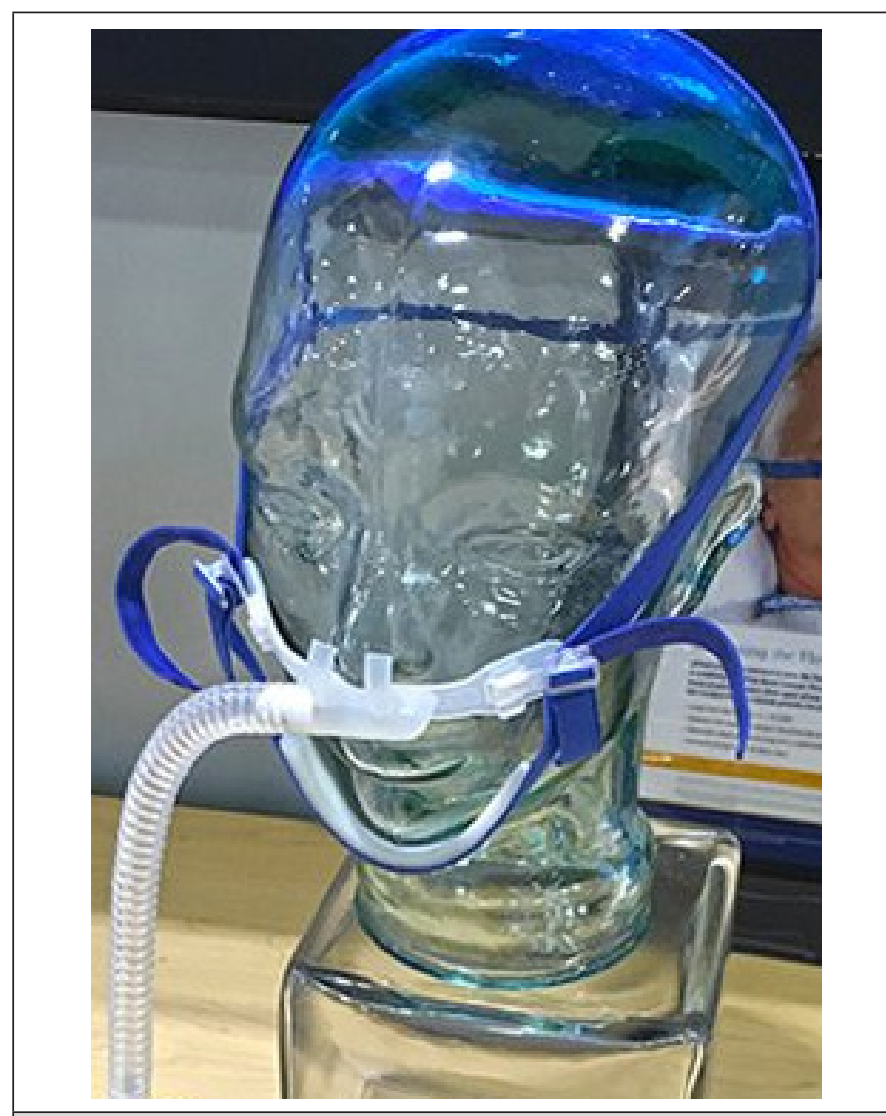

Figure 5A: Face Mask for non-invasive nasal ventilation in severe acute hypoxemic respiratory failure of Covid patients.

The high flow rates of HFNC and Facemask are likely to increase virus aerosolization. The Helmet device can limit virus spread into the ambient air; it is a reusable single patient interface made of a clear plastic hood on a hard-plastic ring with a silicon-polyvinyl chloride soft collar. With an additional tool, the patient's exhalate can be filtered by applying a high efficiency particulate (HEPA) filter at the Helmet outlet [56,57] (Figure 5B). 


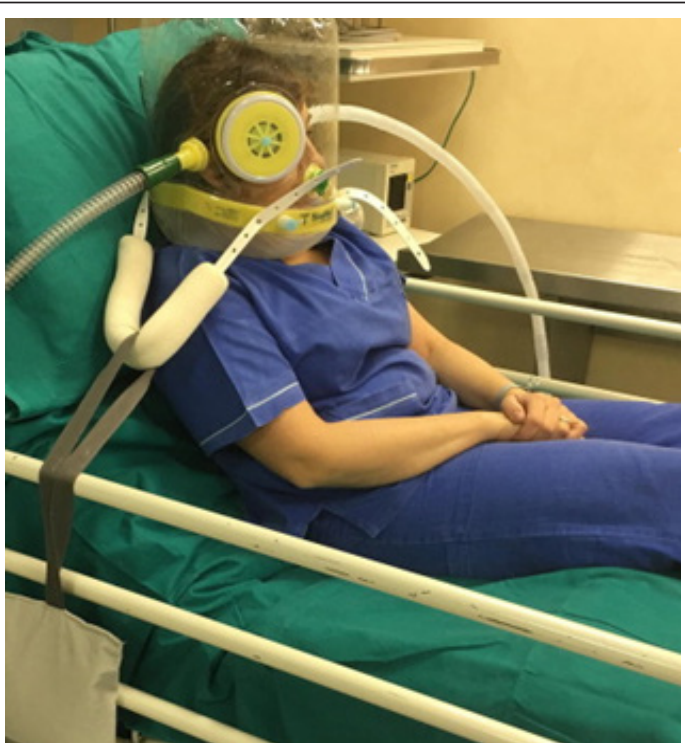

Figure 5B: Helmet non-invasive ventilation device to limit virus spread into the ambient air. Additional filter in the outlet circuit.

\section{Therapeutic Plasma Exchange (TPE)}

It is clinically estimated that $13 \%$ of Covid-19 cases are severe, and $6 \%$ are critical; the evolution can be acute respiratory distress syndrome (ARDS), sepsis and /or multiorgan failure. The response to fulminant COVID-19 infection is characterized by excessive immune dysregulation (cytokine storm), inflammation, hypercoagulable state, and endothelial dysfunction. Severe COVID-19 disease has been associated with lymphopenia and high levels of ferritin, $\mathrm{C}$ reactive protein (CRP), lactate dehydrogenase (LDH), D-dimer and interleukin-6 (IL-6) [4,58,59].

Therapeutic plasma exchange has been used for the management of severe infections such as 2009 HIN1 influenza A, sepsis and multiorgan failure with a trend towards improved survival. In patients with COVID-19 pneumonia, high risk of thrombosis became a current issue, and D-dimer levels indicating fibrin degradation products (FDPs) in the plasma were found as a predictor for mortality $[60,61]$. Although unfractionated heparin (UFH) and low-molecular-weight heparin (LMWH) decrease the production of FDPs by inhibiting factors Xa and II, they cannot contribute metabolization of existing FDPs. Furthermore, FDPs cannot be filtered by known cytokine filters because of their molecular weight (minimum $240 \mathrm{kDa}$ ). FDPs can be removed by therapeutic plasma exchange. TPE might be proposed as supportive/adjunct therapy for the management of COVID-19 with severe pneumonia, cytokine storm, and multiorgan failure $[62,63]$.

\section{Structures of Coronaviral Proteins by Synchrotron Ra- diation Diffraction (Polytechnic University of Ancona, Italy)}

Some determinations of 3D structures at atomic level of proteins and complexes, useful for discovery of drugs and vaccines, were performed by using the diffraction of X-Ray Synchrotron Radiation (64). Synchrotron light is created when centripetal acceleration is applied to an electron beam (Figure 6A). The electrons inside a heated metal are excited enough to escape from the surface in a process known as thermionic emission, then they are directed by the linear accelerator or LINAC. This fits into the Booster ring, which adopts magnetic fields to force the electrons to travel in a circle. Microwaves are used to add even more energy to the electrons. The radiation is produced in the Booster ring, having a multi-sided shape (similar an octagon). A charged particle in motion submitted to a magnetic field perpendicular to its trajectory experiences a force. If the field is constant, the particle describes a circular motion (Figure 6B).

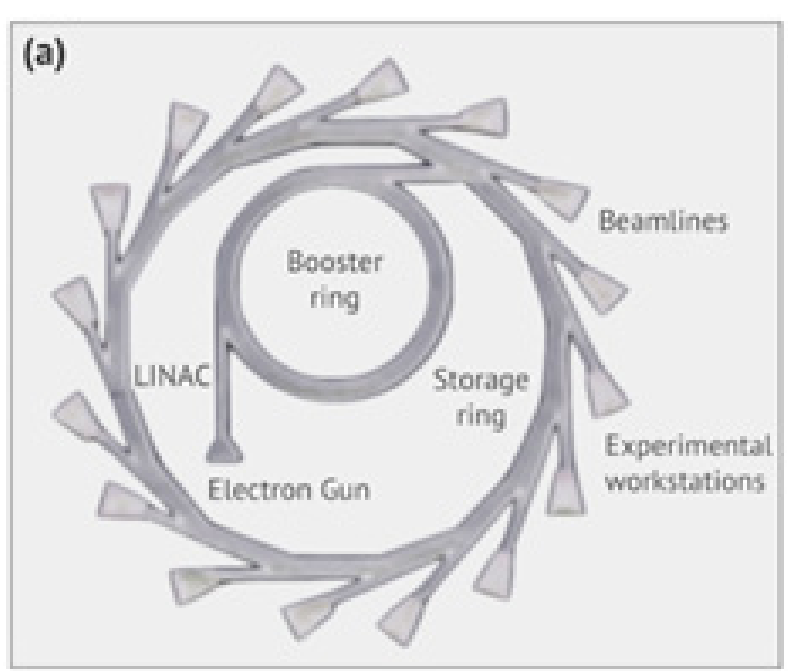

(b)

Figure 6: Synchrotron radiation technique: (a) schematic representation of a synchrotron; (b) a charged particle in motion subjected to a magnetic field perpendicular to its trajectory experiences a force. If the field is constant, the particle describes a circular motion. 
A series of electromagnetic devices (dipole and wave) are positioned around the storage ring, causing the beam to curve or wave in a winding path. Every time the beam passes through a corner with these magnets, the electrons lose energy, which is released in the form of light. These magnets are adjustable. If the intensity of the magnetic field increases, the forces in the electron stream increase, creating tighter curves along its path. This change in the curve produces a modification in the wavelength of the emitted radiation. A tight curve produces short wavelength radiation, like X-rays. While smooth curves produce longer wavelength radiation, such as infrared (Protein crystallography at BESSY II: A mighty tool for the search of anti-viral agents).

The crystal structure of SARS-COV-2 main protease has been determined $[65,66]$. Such an enzyme constitutes a possible target of antiviral drugs because it is essential for processing the polyproteins which are translated from the viral DNA. Then the 3D structure at the atomic level of the main protease complexed with an $\alpha$-ketoamide inhibitor was determined including positive pharmacokinetic results obtained in CD-1 mice. The structure of SARS$\mathrm{CoV}-2$ papain-like protease was also investigated to study mechanism and inhibition [67].

The same technique was used at the Advanced Photon Source of the Argonne National Laboratory (Chicago, USA) to determine the crystal structure of the SARS-CoV-2 receptor binding domain (RBD) in complex with the human cell receptor, namely angiotensin-converting enzyme 2 (ACE 2). A similar experiment was performed at the Synchrotron Research Facility (Shanghai, China).

The crystal structure of baicalein-bound SARS-CoV-2 3CLpro was investigated, demonstrating the 3CLpro reversibly inhibited by a small molecule, or even a fragment of one, derived from TCM, providing an ideal lead for the design of new and versatile classes of inhibitors against 3CLpros [68].

The crystal structure of the C-terminal domain of SARS-CoV-2 (SARS-CoV-2-CTD) spike (S) protein in complex with human ACE2 (hACE2) was also investigated, which reveals a hACE2-binding mode similar overall to that observed for SARS-CoV. The results shed light on the viral pathogenesis, providing important structural information to develop therapeutic countermeasures against the virus [69].

The crystal structure of the receptor-binding domain (RBD) of the spike protein of SARS-CoV-2 bound to the cell receptor ACE2 was also investigated, identifying residues in the SARS-CoV-2 RBD that are essential for ACE2 binding and helping the future identification of cross-reactive antibodies [70].

The above reported results of the different experiments constitute a fundamental target for antiviral strategies, together with the use of neutralizing monoclonal antibodies.

\section{Discussion}

Covid-19 is a prothrombotic disease with a unique global lethality. Strong inflammatory response to viral infection severely affects the respiratory, cardiovascular, immune, and hematologic systems. The coronavirus can send the body's immune system into cataclysmic overdrive, resulting from "cytokine storm syndrome" [71].

Early signs and symptoms associated with COVID-19 infection are skin vasculitis lesions represented by acro-ischemia syndrome of paramount importance for diagnostic in child and adults. These skin complications can be localized in toes, heel and sole of the foot, face, ears, nose, hands, fingers, they can be dangerous signs of disseminated intravascular coagulation with mortality risks [35-38]. They are mostly related with immune dysregulation, neoangiogenesis, vasculitis, and vessel thrombosis. Fast identification of acro-ischemia syndrome permits the care of disseminated intravascular coagulation complications. Furthermore, neuro-sensorial symptoms like olfactory and gustatory loss (anosmia, dysgeusia) are also initial symptoms useful for covid diagnosis and patients' isolation [46-48]. The goal is avoiding epidemic spreading.

Clinical evolution of patients allows to consider COVID-19 as a new hematologic disease [72]. Curative anticoagulation in these patients prevents endothelial lesions. Angiopoietin-2 as a marker of endothelial activation is a good predictor factor for intensive care unit admission of COVID-19 patients [25-27]. Coronavirus antibodies can disappear after 2-3 months of disease. Assessment of antibodies levels after recovery showed that patients cured of SARS-CoV-2 are only immunized for several weeks, thus antibodies remain in patient's serum for at least 40 days after the onset of signs. These antibodies persist active and dynamic to prevent reinfection for at least three months in $90 \%$ of cases [73]. This rate begins to decline after twenty to thirty days, most subjects tested lose $50 \%$ of active antibodies after six months. Afterwards lymphocytes develop and take in charge immunity, the so-called "memory" cells in turn would be ready to produce antibodies in the event of recontamination. Further research should clarify this subject. Exosomes could be a therapeutic option in coronavirus disease [74,71].

Mathematical models address the need for understanding the transmission dynamics and other significant factors of the disease that would aid policymakers to make accurate decisions and reduce the rate of transmission of the disease. Synchrotron radiation might play a role for diagnostic and therapy. Aftermath of Covid-19 is for the moment unknown; cardiovascular, hematological, neurological, respiratory, and immune systems are greatly altered [76-78]. Future translational research should be concentrated in this challenge. 


\section{Abbreviations}

COVID

: Corona Virus Disease

SARS CoV

Syndrome Coronavirus

: Severe Acute Respiratory

ECMO

Oxygenation

ACE

zyme

RT-PCR

Chain Reaction

PEEP

Pressure

ARDS

Syndrome

HFNC

NIV

$\mathrm{L} / \min$

HEPA

filter

CMR

nance

DVT

MERS-CoV

Syndrome Coronavirus

D-DIMER

ICU

DIC

Coagulation

CNS

CT

LU

SEIR Epidemiology model

tious-Recovered

\section{Declarations}

Competing interests: The authors declare that they have no competing interests.

Financial Disclosure statement: The authors received no specific funding for this work.

\section{Authors contributions}

Conceived and designed the study: JCC, DM, FR. Search of information and database creation: JCC, LM, CH, BZ, MR. Analyzed the data: JCC, LM, CH, CL. Clinical and epidemiological explorations: JCC, DM, LM, CL. Contributed documentation \& illustrations: JCC, LM, BZ, MR, CL. Wrote the paper: JCC LM CH DM BZ MR FR. Compiled the data: DM, CH, FR.

\section{Acknowledgement}

The authors acknowledge networking support by the European Union COST Action CA16122.

\section{References}

1. Zhu N, Zhang D, Wang W, Li X, Yang B, et al. (2020) A novel coronavirus from patients with pneumonia in China, 2019. N Engl J Med 382: 727733.

1. Liu X, Yue X, Liu F, Wei L, Chu Y, et al. (2020) Analysis of clinical features and early warning signs in patients with severe COVID-19: A retrospective cohort study. PLoS One 15(6): e0235459.

2. Stein RA, Young LM (2020) From ACE2 to COVID-19: A multiorgan endothelial disease. Int J Infect Dis 100: P425-P430.

3. Huang Y, Lyu X, Li D, Wang L, Wang Y, et al. (2020) A cohort study of 676 patients indicates D-dimer is a critical risk factor for the mortality of COVID-19. PLoS One 15(11): e0242045.

4. Liu JL, Khawaja AM, Majjhoo AQ (2020) Stroke as a delayed manifestation of multi-organ thromboembolic disease in COVID-19 infection. J Neurol Sci 417: 117071.

5. Wu Z, Mc Googan JM (2020) Characteristics of and important lessons from the coronavirus disease 2019 (COVID-19) outbreak in China: Summary of a report of 72,314 cases from the Chinese center for disease control and prevention. JAMA 323(13): 1239-1242.

6. Akhmerov A, Marban E (2020) COVID-19 and the Heart. Circ Res 126(10): 1443-1455.

7. Lescure FX, Bouadma L, Nguyen D, Parisey M, Wicky PH, et al. (2020) Clinical and virological data of the first cases of COVID-19 in Europe: a case series. Lancet Infect Dis 20(6): 697-706.

8. Lagier D, Fischer F, Fornier W, Huynh TM, Cholley B, et al. (2019) PROVECS Study Group. Effect of open-lung vs conventional perioperative ventilation strategies on postoperative pulmonary complications after on-pump cardiac surgery: the PROVECS randomized clinical trial. Intensive Care Med 45(10): 1401-1412.

9. Richardson S, Hirsch JS, Narasimhan M, Crawford JM, McGinn T, et al. (2020) Presenting characteristics, comorbidities, and outcomes among 5700 patients hospitalized with COVID-19 in the New York City Area. JAMA 323(20): 2052-2059.

10. Ackermann M, Verleden SE, Kuehnel M, Haverich A, Welte T, et al. (2020) Pulmonary vascular endothelialitis, thrombosis, and angiogenesis in Covid-19. N Engl J Med 383(2): 120-128.

11. Arachchillage DJ, Stacey A, Akor F, Scotz M, Laffan M, et al. (2020) Thrombolysis restores perfusion in COVID-19 hypoxia. Br J Haematol 190(5): e270-e274.

12. Dolhnikoff M, Duarte Neto AN, de Almeida Monteiro RA (2020) Pathological evidence of pulmonary thrombotic phenomena in severe COVID-19. J Thromb Haemost 18(6): 1527-1529. 
13. Ai T, Yang Z, Hou H, Zhan C, Chen C, et al. (2020) Correlation of chest CT and RT-PCR testing for coronavirus disease 2019 (COVID-19) in China: A report of 1014 cases. Radiology 296(2): E32-E40.

14. Vetrugno L, Bove T, Orso D, Barbariol F, Bassi F, et al. (2020) Our Italian experience using lung ultrasound for identification, grading and serial follow-up of severity of lung involvement for management of patients with COVID-19. Echocardiography 37(4): 625-627.

15. Xiong TY, Redwood S, Prendergast B, Chen M (2020) Coronaviruses and the cardiovascular system: acute and long-term implications. Eur Heart J 41(19): 1798-1800

16. Long B, Brady WJ, Koyfman A, Gottlieb M (2020) cardiovascular complications in COVID-19. Am J Emerg Med 38(7): 1504-1507.

17. Puntmann VO, Carerj ML, Wieters I, Fahim M, Arendt C, et al. (2020) Outcomes of cardiovascular magnetic resonance imaging in patients recently recovered from coronavirus disease 2019 (COVID-19). JAMA Cardiol 5(11): 1265-1273.

18. Olejniczak M, Schwartz M, Webber E, Shaffer A, Perry TE, et al. (2020) Viral myocarditis - Incidence, Diagnosis and Management. J Cardiothorac Vasc Anesth 34(6): 1591-1601.

19. Ho JS, Sia CH, Chan MY, Lin W, Wong RC, et al. (2020) Coronavirus-induced myocarditis: A meta-summary of cases. Heart Lung 49(6): 681685.

20. Hekimian G, Jovanovic T, Brechot N, Lebreton G, Leprince P, et al.(2018) When the heart gets the flu: Fulminant influenza B myocarditis: A case-series report and review of the literature. J Crit Care 47: 61-64.

21. Chachques JC, Jegaden O, Mesana T, Glock Y, Grandjean PA, et al.(2009) French Cardiomyoplasty Investigators. Cardiac bioassist: results of the French multicenter cardiomyoplasty study. Asian Cardiovasc Thorac Ann 17(6): 573-580.

22. Herreros J, Trainini JC, Chachques JC (2011) Alternatives to heart transplantation: integration of biology with surgery. Front Biosci (Elite Ed) 3: 635-647.

23. Chachques JC, Jegaden OJ, Bors V, Mesana T, Latremouille C, et al.(2008) Heart transplantation following cardiomyoplasty: a biological bridge. Eur J Cardiothorac Surg 33(4): 685-690.

24. Debuc B, Smadja DM (2020) Is COVID-19 a new hematologic disease? Stem Cell Rev Rep 12: 1-5.

25. Khider L, Gendron N, Goudot G, Chocron R, Hauw Berlemont C, et al. (2020) Curative anticoagulation prevents endothelial lesion in COVID-19 patients. J Thromb Haemost.

26. Smadja DM, Guerin CL, Chocron R, Yatim N, Boussier J, et al. (2020) Angiopoietin-2 as a marker of endothelial activation is a good predictor factor for intensive care unit admission of COVID-19 patients. Angiogenesis 23(4): 611-620.

27. Connors JM, Levy JH (2020) COVID-19 and its implications for thrombosis and anticoagulation. Blood 135(23): 2033-2040.

28. Zhang L, Feng X, Zhang D, Jiang C, Mei H, et al. (2020) Deep vein thrombosis in hospitalized patients with COVID-19 in Wuhan, China: Prevalence, risk factors, and outcome. Circulation 142(2): 114-128.

29. Carsana L, Sonzogni, Nasr A, Rossi RS, Pellegrinelli A, et al. (2020) pulmonary post-mortem findings in a large series of COVID-19 cases from Northern Italy: a two-centre descriptive study. Lancet Infect Dis 20(10): 1135-1140.

30. Vasquez Bonilla WO, Orozco R, Argueta V, Sierra M, Zambrano LI, et al. (2020) A review of the main histopathological findings in the Coronavirus Disease 2019 (COVID-19). Hum Pathol 105: 74-83.

31. Jacobs JP, Stammers AH, St Louis J, Awori Hayanga JW, Firstenberg MS, et al. (2020) extracorporeal membrane oxygenation in the treatment of severe pulmonary and cardiac compromise in COVID-19: Experience with 32 patients. ASAIO J 66(7): 722-730.
32. Le Gall A, Follin A, Cholley B, Mantz J, Aissaoui N, et al. (2018) Veno-arterial-ECMO in the intensive care unit: From technical aspects to clinical practice. Anaesth Crit Care Pain Med 37(3): 259-268.

33. Ortuno S, Delmas C, Diehl JL, Bailleul C, Lancelot A, et al. (2019) Weaning from veno-arterial extra-corporeal membrane oxygenation: which strategy to use? Ann Cardiothorac Surg 8(1): E1-E8.

34. Günther C, Aschoff R, Beissert S (2020) cutaneous autoimmune diseases during COVID 19 pandemic. J Eur Acad Dermatol Venereol.

35. Wollina U, Karadağ AS, Rowland Payne C, Chiriac A, Lotti T, et al. (2020) cutaneous signs in COVID-19 patients: A review. Dermatol Ther 10: e13549.

36. Cordoro KM, Reynolds SD, Wattier R, McCalmont TH (2020) Clustered cases of acral perniosis: Clinical features, histopathology and relationship to COVID-19. Pediatr Dermatol 37(3): 419-423.

37. Mayor Ibarguren A, Feito Rodriguez M, Quintana Castanedo L, Ruiz Bravo E, Montero Vega D, et al. (2020) Cutaneous small vessel vasculitis secondary to COVID-19 infection. J Eur Acad Dermatol Venereol 34(10): e541-e542.

38. Mao L, Jin H, Wang M, Hu Y, Chen S, et al. (2020) Neurologic manifestations of hospitalized patients with coronavirus disease 2019 in Wuhan, China. JAMA Neurol 77(6): 683-690.

39. Asadi Pooya AA, Simani L (2020) Central nervous system manifestations of COVID-19: A systematic review, J Neurol Sci 413: 116832.

40. Li YC, Bai WZ, Hashikawa T (2020) the neuroinvasive potential of SARSCoV2 may play a role in the respiratory failure of COVID-19 patients. J Med Virol 92(6): 552-555.

41. Zhou L, Zhang M, Wang J, Gao J (2020) Sars-Cov-2: Underestimated damage to nervous system. Travel Med Infect Dis 36: 101642.

42. Liotta EM, Batra A, Clark JR, Shlobin NA, Hoffman SC, et al. (2020) Frequent neurologic manifestations and encephalopathy-associated morbidity in Covid-19 patients. Ann Clin Transl Neurol 7(11): 2221-2230.

43. Nasuelli NA, Pettinaroli R, Godi L, Savoini C, De Marchi F, et al. (2020) Critical illness neuro-myopathy (CINM) and focal amyotrophy in intensive care unit (ICU) patients with SARS-CoV-2: a case series. Neurol Sci 13: $1-3$.

44. De Marchi F, Cantello R, Ambrosini S, Mazzini L (2020) CANPALS Study Group. Telemedicine and technological devices for amyotrophic lateral sclerosis in the era of COVID-19. Neurol Sci 41(6): 1365-1367.

45. Lechien JR, Chiesa Estomba CM, De Siati DR, Horoi M, Le Bon SD, et al. (2020) Olfactory and gustatory dysfunctions as a clinical presentation of mild-to-moderate forms of the coronavirus disease (COVID-19): a multicenter European study. Eur Arch Otorhinolaryngol 277(8): 2251-2261.

46. Butowt R, Bilinska K (2020) SARS-CoV-2: Olfaction, brain Infection, and the urgent need for clinical samples allowing earlier virus detection. ACS Chem Neurosci 11: 1200-1203.

47. Gane SB, Kelly C, Hopkins C (2020) Isolated sudden onset anosmia in COVID-19 infection. A novel syndrome? Rhinology 58(3): 299-301.

48. Hellewell J, Abbott S, Gimma A, Bosse NI, Jarvis CI, et al.(2020) Centre for the Mathematical Modelling of Infectious Diseases COVID-19 Working Group, Funk S, Eggo RM. Feasibility of controlling COVID-19 outbreaks by isolation of cases and contacts. Lancet Glob Health 8(4): e488-e496.

49. Roda WC, Varughese MB, Han D, Li MY (2020) Why is it difficult to accurately predict the COVID-19 epidemic? Infectious Disease Modelling 5: 271-281.

50. Pastrama MI, Scheiner S, Pivonka P, Hellmich C (2018) Mathematical multiscale model of bone remodeling, accounting for pore space-specific mechanosensation. Bone 107: 208-221. 
51. Scheiner S, Ukaj N, Hellmich C (2020) Mathematical modeling of COVID-19 fatality trends: Death kinetics law versus infection-to-death delay rule. Chaos Solitons Fractals 136: 109891.

52. Truelove S, Abrahim O, Altare C, Lauer SA, Grantz KH, et al. (2020) the potential impact of COVID-19 in refugee camps in Bangladesh and beyond: A modeling study. PLoS Med 17(6): e1003144.

53. Guan L, Zhou L, Le Grange JM, Zheng Z, Chen R, et al. (2020) Non-invasive ventilation in the treatment of early hypoxemic respiratory failure caused by COVID-19: considering nasal CPAP as the first choice. Crit Care 24(1): 333

54. Demoule A, Vieillard Baron A, Darmon M, Beurton A, Géri G, et al. (2020) High-Flow nasal cannula in critically iII patients with severe COVID-19. Am J Respir Crit Care Med 202(7): 1039-1042.

55. Cabrini L, Landoni G, Zangrillo A (2020) Minimise nosocomial spread of 2019-nCoV when treating acute respiratory failure. Lancet 395(10225): 685.

56. Lucchini A, Giani M, Isgro S, Rona R, Foti G, et al. (2020) The "helmet bundle" in COVID-19 patients undergoing non-invasive ventilation. Intensive Crit Care Nurs 58: 102859.

57. Helms J, Tacquard C, Severac F, Leonard Lorant I, Ohana M, et al. (2020) High risk of thrombosis in patients with severe SARS-CoV-2 infection: a multicenter prospective cohort study. Intensive Care Med 46(6):10891098.

58. Herold T, Jurinovic V, Arnreich C, Lipworth BJ, Hellmuth JC, et al. (2020) Elevated levels of IL- 6 and CRP predict the need for mechanical ventilation in COVID-19. J Allergy Clin Immunol 146(1): 128-136.

59. Khamis F, Al Zakwani I, Al Hashmi S, Al Dowaiki S, Al Bahrani M, et al. (2020) Therapeutic plasma exchange in adults with severe COVID-19 infection. Int J Infect Dis 99: 214-218.

60. Gucyetmez B, Atalan HK, Sertdemir I, Cakir U, Telci L, et al. (2020) COVID-19 Study Group. Therapeutic plasma exchange in patients with COVID-19 pneumonia in intensive care unit: a retrospective study. Crit Care 24(1): 492.

61. Tabibi S, Tabibi T, Conic RRZ, Banisaeed N, Streiff MB, et al. (2020) Therapeutic plasma exchange: A potential management strategy for critically Ill COVID-19 patients. J Intensive Care Med 35(9): 827-835.

62. Ma J, Xia P, Zhou Y, Liu Z, Zhou X, et al. (2020) Potential effect of blood purification therapy in reducing cytokine storm as a late complication of critically ill COVID-19. Clin Immunol 214: 108408.

63. Mangano C, Piattelli A, Mangano F, Rustichelli F, Shibli JA, et al. (2013) Histological and synchrotron radiation-based computed microtomography study of 2 human-retrieved direct laser metal formed titanium implants. Implant Dent 22(2): 175-181
64. Zhang L, Lin D, Kusov Y, Nian Y, Ma Q, et al. (2020) $\alpha$-Ketoamides as broad-spectrum inhibitors of coronavirus and enterovirus replication: structure-based design, synthesis, and activity assessment. J Med Chem 63(9): 4562-4578.

65. Zhang L, Lin D, Sun X, Curth U, Drosten C, et al. (2020) Crystal structure of SARS-CoV-2 main protease provides a basis for design of improved $\alpha$-ketoamide inhibitors. Science 368(6489): 409-412.

66. Klemm T, Ebert G, Calleja DJ, Allison CC, Richardson LW, et al. (2020) Mechanism and inhibition of the papain-like protease, PLpro, of SARSCoV-2. EMBO J 39(18): e106275.

67. Su HX, Yao S, Zhao WF, Li MJ, Liu J, et al. (2020) Anti-SARS-CoV-2 activities in vitro of Shuanghuanglian preparations and bioactive ingredients. Acta Pharmacol Sin 41(9): 1167-1177.

68. Wang Q Zhang Y, Wu L, Niu S, Song C, et al. (2020) Structural and functional basis of SARS-CoV-2 entry by using human ACE2. Cell 181(4): 894904.

69. Lan J, Ge J, Yu J, Shan S, Zhou H, et al. (2020) Structure of the SARS-CoV-2 spike receptor-binding domain bound to the ACE2 receptor. Nature 581(7807): 215-220.

70. Mangalmurti N, Hunter CA (2020) Cytokine Storms: Understanding COVID-19. Immunity 53(1): 19-25.

71. Becker RC (2020) Covid-19-associated coagulopathy. J Thromb Thrombolysis 50(1): 54-67.

72. Seow J, Graham C, Merrick B, Acors S, Pickering S, et al. (2020) Longitudinal observation and decline of neutralizing antibody responses in the three months following SARS-CoV-2 infection in humans. Nat Microbiol.

73. Bellin G, Gardin C, Ferroni L, Chachques JC, Rogante M, et al. (2020) Exosome in cardiovascular diseases: A complex world full of hope. Cells 8(2): 166

74. Cardin C, Ferroni L, Chachques JC, Zavan B (2020) could mesenchymal stem cells-derived exosomes be a therapeutic option for critically ill COVID-19 patients? J Clin Med 9(9): 2762.

75. (2020) COVID Surg Collaborative. Mortality and pulmonary complications in patients undergoing surgery with perioperative SARS-CoV-2 Infection: An international cohort study. Lancet 396(10243): 27-38.

76. Mueller AA, Tamura T, Crowley CP, DeGrado JR, Haider H, et al.(2020) Inflammatory biomarker trends predict respiratory decline in COVID-19 patients. Cell Rep Med 1(8): 100144.

77. Hoiland RL, Fergusson NA, Mitra AR, Griesdale DEG, Devine DV, et al (2020) The association of ABO blood group with indices of disease severity and multiorgan dysfunction in COVID-19. Blood Adv 4(20): 49814989. 